\title{
A Class of Beta Second Kind Mixture Distributions
}

\author{
Mian Arif Shams Adnan ${ }^{1, *,([), ~ H u m a y u n ~ K i s e r ~}{ }^{2}$ \\ ${ }^{1}$ Department of Mathematics and Statistics, Bowling Green State University, Bowling Green, Ohio, 43402, USA \\ ${ }^{2}$ School of Mathematical Sciences, University of Nottingham, University Park, Nottingham, NG7 2RD, England
}

\section{ARTICLE INFO}

Article History

Received 21 Dec 2019

Accepted 20 Mar 2020

Keywords

Methods of moments

Mixing distribution

Mixtured distribution

\section{ABSTRACT}

A class of mixture distributions have been derived which we call beta second kind mixtures of distributions. Various integral representations of beta functions can be obtained using these mixture beta distributions. Estimation of unknown parameters along with some characteristics of these distributions are also been found.

(C) 2021 The Authors. Published by Atlantis Press B.V. This is an open access article distributed under the CC BY-NC 4.0 license (http://creativecommons.org/licenses/by-nc/4.0/).

\section{INTRODUCTION}

Mixture distribution [1,2] was first coined in 1894. A number of authors like Pearson [3], Rider [4], Blichke [5-8], Cohen [9], Chahine [10], Hasselblad [11], Day [12], Jewell [13], Roy et al. [14-16], Adnan et al. [17-26] defined mixtures of two distributions and studied various mixtured distributions which they called poisson mixture, binomial mixture, negative binomial mixture, chi-square mixture, erlang mixture, laplace mixture, pareto mixture, $F$ mixture, weibull mixture and Maxwell mixture of distributions. Adnan et al. [27,28] also studied several properties of triple and folded Gamma mixture distributions.

\section{PRELIMINARIES}

A mixture distribution is a weighted average of probability distribution of positive weights that sum to one. The weights themselves constitutes a probability distribution. The mixing distribution $g(x ; \theta)$ is a weighted average of a distribution in either in $\sum_{i=1}^{k} f\left(x ; \theta_{i}\right) g\left(\theta_{i}\right)$ form or in $\int f(x ; \theta) g(\theta) d \theta$ form.

\section{MAIN RESULTS}

Here in this paper, we define the beta second kind mixtures of some well-known distributions such as normal, lognormal, gamma, exponential, beta second kind, rectangular, erlang, chi-square, $t$, and $F$ distributions. Then some characteristics of these distributions such as characteristic functions, moments, and shape characteristics are also obtained. The main results of the paper are presented in form of definitions and theorems.

Definition 3.1. A random variable $\mathrm{X}$ is said to have a beta second kind mixtured distribution if its probability density function is defined as

$$
f(x ; p, q, \alpha)=\int_{0}^{\infty} \frac{1}{B(p, q)} \frac{r^{p-1}}{(1+r)^{p+q}} g(x ; \alpha) d r
$$

where $g(x ; \alpha)$ is a probability density function. The name of second kind mixture distribution comes from the fact that the distribution (1) is the weighted average of $g(x ; \alpha)$ with weights equal to the ordinates of second kind distribution. 
Definition 3.2. If $\mathrm{X}$ follows a beta second kind mixture of normal distribution with parameters $p$ and $q$, then the density function is given by

$$
f(x ; p, q)=\int_{0}^{\infty} \frac{1}{B(p, q)} \frac{r^{p-1}}{(1+r)^{p+q}} \frac{e^{-\frac{1}{2} x^{2}} x^{2 r}}{2^{\mathrm{r}+\frac{1}{2}} \Gamma\left(\mathrm{r}+\frac{1}{2}\right)} d r ;-\infty<x<\infty
$$

with parameters $p$ and $q$ since

$$
\int_{-\infty}^{\infty} f(x ; p, q) d x=1
$$

The characteristic function and moments of the same distribution are presented in the theorem below.

Theorem 3.1. If X has a beta second kind mixture of normal distributions with parameters $p$ and $q$ then its characteristic function is represented as

$$
\int_{0}^{\infty} \frac{1}{B(p, q)} \frac{r^{p-1}}{(1+r)^{p+q}} \frac{e^{-\frac{1}{2} t^{2}}}{2^{r+\frac{1}{2}} \Gamma\left(r+\frac{1}{2}\right)} \sum_{m=0}^{r}\left(\begin{array}{c}
2 r \\
2 m
\end{array}\right)(i t)^{2 m} 2^{r+\frac{1}{2}-m} \Gamma\left(r+\frac{1}{2}-m\right) d r
$$

and the $2 s^{\text {th }}$ moment about origin is $\int_{0}^{\infty} \frac{1}{B(p, q)} \frac{r^{p-1}}{(1+r)^{p+q}} 2^{s} \frac{\Gamma\left(r+\frac{1}{2}+s\right)}{\Gamma\left(r+\frac{1}{2}\right)} d r$ and $(2 s+1)^{\text {th }}$ moment about origin is zero. Mean $=0$, Variance $=1+2 \frac{p}{q-1}$,

$$
\beta_{1}=0, \quad \beta_{2}=\frac{\left[3+8 \frac{p}{q-1}+4 \frac{p(p+1)}{(q-1)(q-2)}\right]}{\left[1+2 \frac{p}{q-1}\right]^{2}}
$$

Remark. If $p=q=0$ then all the values of $\phi_{x}(t), \mu_{2 s+1}^{\prime}, \mu_{2 s}^{\prime}, \mu_{1}, \mu_{2}, \mu_{3}, \mu_{4}, \beta_{1}$ and $\beta_{2}$ are true for normal distribution with mean zero and variance unity.

Definition 3.3. If a random variable $\mathrm{X}$ has the density function

$$
f(x ; p, q)=\int_{0}^{\infty} \frac{1}{B(p, q)} \frac{r^{p-1}}{(1+r)^{p+q}} \frac{e^{-\frac{1}{2}(\log x)^{2}}(\log x)^{2 r}}{x 2^{\mathrm{r}+\frac{1}{2}} \Gamma\left(\mathrm{r}+\frac{1}{2}\right)} d r ; x>0
$$

then it is said to have a beta second kind mixture of lognormal distribution with parameter $p$ and $q$ since

$$
\int_{0}^{\infty} f(x ; p, q) d x=1
$$

Various moments of the distribution are given in the next theorem.

Theorem 3.2. If $X$ is a beta second kind mixture of lognormal variable with parameters $p$ and $q$ then its characteristic function is given by

$$
\int_{0}^{\infty} \frac{1}{B(p, q)} \frac{r^{p-1}}{(1+r)^{p+q}} \frac{1}{2^{r+\frac{1}{2}} \Gamma\left(r+\frac{1}{2}\right)} \sum_{k=0}^{\infty} \frac{(i t)^{k}}{k !} e^{\frac{1}{2} k^{2}} \sum_{m=0}^{r}\left(\begin{array}{c}
2 r \\
2 m
\end{array}\right) k^{2 r-2 m} 2^{m+\frac{1}{2}} \Gamma\left(m+\frac{1}{2}\right) d r
$$

and the $s^{\text {th }}$ moment about origin is

$$
\mu_{s}^{\prime}=\int_{0}^{\infty} \frac{1}{B(p, q)} \frac{r^{p-1}}{(1+r)^{p+q}} \frac{e^{\frac{1}{2} s^{2}}}{2^{r-m} \Gamma\left(r+\frac{1}{2}\right)} \sum_{m=0}^{r}\left(\begin{array}{c}
2 r \\
2 m
\end{array}\right) s^{2 r-2 m} \Gamma\left(m+\frac{1}{2}\right) d r
$$

Definition 3.4. A random variable $\mathrm{X}$ having the density function

$$
f(x ; p, q, \alpha, \beta)=\int_{0}^{\infty} \frac{1}{B(p, q)} \frac{r^{p-1}}{(1+r)^{p+q}} \frac{\beta^{\alpha+r} e^{-\beta x} x^{\alpha+r-1}}{\Gamma(\alpha+\mathrm{r})} d r ; x>0,
$$

is defined a beta second kind mixture of Gamma distribution with parameters $p, q, \alpha$ and $\beta$ since

$$
\int_{0}^{\infty} f(x ; p, q, \alpha, \beta) d x=1 .
$$

The characteristic function and moments are provided in the theorem below. 
Theorem 3.3. If $X$ denotes a beta second kind mixture of gamma variate with parameters $p, q, \alpha$ and $\beta$ then its characteristic function is obtained as

$$
\frac{1}{B(p, q)}\left(1-\frac{i t}{\beta}\right)^{-\alpha} \int_{0}^{\infty} \frac{r^{p-1}}{(1+r)^{p+q}} e^{-r \ln \left(1-\frac{i t}{\beta}\right)} d r
$$

and Mean $=\frac{1}{\beta}\left[\alpha+\frac{p}{q-1}\right]$, Variance $=\frac{1}{\beta^{2}}\left[\alpha+\frac{p}{q-1}+\frac{p(p+1)}{(q-1)(q-2)}-\frac{p^{2}}{(q-1)^{2}}\right]$,

$$
\begin{gathered}
\beta_{1}=\frac{\left[2 \alpha+2 \frac{p}{q-1}+3 \frac{p(p+1)}{(q-1)(q-2)}+\frac{p(p+1)(p+2)}{(q-1)(q-2)(q-3)}-3 \frac{p^{2}}{(q-1)^{2}}-3 \frac{p^{2}(p+1)}{(q-1)^{2}(q-2)}+2 \frac{p^{3}}{(q-1)^{3}}\right]^{2}}{\left[\alpha+\frac{p}{q-1}+\frac{p(p+1)}{(q-1)(q-2)}-\frac{p^{2}}{(q-1)^{2}}\right]^{3}}, \\
\beta_{2}=\frac{\left.-(6 \alpha+8) \frac{p^{2}}{(q-1)^{2}}-12 \frac{p^{2}(p+1)}{(q-1)^{2}(q-2)}-4 \frac{p^{2}(p+1)(p+2)}{(q-1)^{2}(q-2)(q-3)}+6 \frac{p^{3}}{(q-1)^{3}}+6 \frac{p^{3}(p+1)}{(q-1)^{3}(q-2)}-3 \frac{p^{4}}{(q-1)^{4}}\right]}{\left[\alpha+\frac{p}{q-1}+\frac{p(p+1)}{(q-1)(q-2)}-\frac{p^{2}}{(q-1)^{2}}\right]^{2}} .
\end{gathered}
$$

Remark. If $p=q=0$ then all the values of $\phi_{x}(t), \mu_{s}^{\prime}, \mu_{1}, \mu_{2}, \mu_{3}, \mu_{4}, \beta_{1}$ and $\beta_{2}$ are true for Gamma distribution with parameters $\alpha$ and $\beta$.

Estimates of parameters by the method of moments: Let $X_{1}, X_{2}, \ldots . . X_{m}$ be a random sample from the distribution (8). We assume that parameters $p, q$ and $\beta$ are known. Then the distribution contains only one unknown parameter $\alpha$. We have $\mu_{1}^{\prime}=\frac{1}{\beta}\left[\alpha+\frac{p}{q-1}\right]$ and $m_{1}^{\prime}=\frac{\sum x_{i}}{m}=\bar{X}$. Hence by the method of moments, we get $\frac{1}{\beta}\left[\alpha+\frac{p}{q-1}\right]=\bar{X}$. Therefore,

$$
\hat{\alpha}=\bar{X} \beta-\frac{p}{q-1}
$$

Definition 3.5. A random variable $\mathrm{X}$ having the density function

$$
f(x ; p, q, \alpha)=\int_{0}^{\infty} \frac{1}{B(p, q)} \frac{r^{p-1}}{(1+r)^{p+q}} \frac{\alpha^{r+1} e^{-\alpha x} x^{r}}{\Gamma(\mathrm{r}+1)} d r ; x>0,
$$

is said to have a beta second kind mixture of Exponential distribution with parameters $p, q$ and $\alpha$ since

$$
\int_{0}^{\infty} f(x ; p, q, \alpha) d x=1
$$

Various characteristics of the above distribution are described in the following theorem.

Theorem 3.4. If $X$ follows beta second kind mixture of exponential distributions with parameters $p, q$ and $\alpha$ then its characteristic function is given by

$$
\frac{1}{B(p, q)}\left(1-\frac{i t}{\alpha}\right)^{-1} \int_{0}^{\infty} \frac{r^{p-1}}{(1+r)^{p+q}} e^{-r \ln \left(1-\frac{i t}{\alpha}\right)} d r
$$

and Mean $=\frac{1}{\alpha}\left[1+\frac{p}{q-1}\right]$, Variance $=\frac{1}{\alpha^{2}}\left[1+\frac{p}{q-1}+\frac{p(p+1)}{(q-1)(q-2)}-\frac{p^{2}}{(q-1)^{2}}\right]$,

$$
\begin{aligned}
& \beta_{1}=\frac{\left[2+2 \frac{p}{q-1}+3 \frac{p(p+1)}{(q-1)(q-2)}+\frac{p(p+1)(p+2)}{(q-1)(q-2)(q-3)}-3 \frac{p^{2}}{(q-1)^{2}}-3 \frac{p^{2}(p+1)}{(q-1)^{2}(q-2)}+2 \frac{p^{3}}{(q-1)^{3}}\right]^{2}}{\left[1+\frac{p}{q-1}+\frac{p(p+1)}{(q-1)(q-2)}-\frac{p^{2}}{(q-1)^{2}}\right]^{3}}, \\
& \beta_{2}=\frac{\left[9+12 \frac{p}{q-1}+17 \frac{p(p+1)}{(q-1)(q-2)}+6 \frac{p(p+1)(p+2)}{(q-1)(q-2)(q-3)}+\frac{p(p+1)(p+2)(p+3)}{(q-1)(q-2)(q-3)(q-4)}-14 \frac{p^{2}}{(q-1)^{2}}\right.}{\left.-12 \frac{p^{2}(p+1)}{(q-1)^{2}(q-2)}-4 \frac{p^{2}(p+1)(p+2)}{(q-1)^{2}(q-2)(q-3)}+6 \frac{p^{3}}{(q-1)^{3}}+6 \frac{p^{3}(p+1)}{(q-1)^{3}(q-2)}-3 \frac{p^{4}}{(q-1)^{4}}\right]} \\
& {\left[1+\frac{p}{q-1}+\frac{p(p+1)}{(q-1)(q-2)}-\frac{p^{2}}{(q-1)^{2}}\right]^{2}}
\end{aligned}
$$


Remark. If $p=q=0$ then all the values of $\phi_{x}(t), \mu_{s}^{\prime}, \mu_{1}, \mu_{2}, \mu_{3}, \mu_{4}, \beta_{1}$ and $\beta_{2}$ are true for Exponential distribution with parameter $\alpha$.

Method of moments: If $X_{1}, X_{2}, \ldots . . X_{m}$ be a random sample drawn from the distribution (12) and parameter $p, q$ is assumed known, then the distribution contains only one unknown parameter $\alpha$. According to the method of moments, we get $\frac{1}{\alpha}\left[1+\frac{p}{q-1}\right]=\bar{X}$. Therefore,

$$
\hat{\alpha}=\frac{\left[1+\frac{p}{q-1}\right]}{\bar{X}}
$$

Definition 3.6. If a random variable $\mathrm{X}$ has the density function

$$
f(x ; p, q, \alpha, \beta)=\int_{0}^{\infty} \frac{1}{B(p, q)} \frac{r^{p-1}}{(1+r)^{p+q}} \frac{(\alpha \beta)^{\alpha+r} e^{-\alpha \beta x} x^{\alpha+r-1}}{\Gamma(\alpha+\mathrm{r})} d r ; x>0,
$$

then it is said to have a beta second kind mixture of Erlang distribution with parameters $p, q, \alpha$ and $\beta$ since

$$
\int_{0}^{\infty} f(x ; p, q, \alpha, \beta) d x=1
$$

The characteristic function as well as the moments is stated in the following theorem.

Theorem 3.5. If $X$ has beta second kind mixture of erlang distributions with parameters $p, q, \alpha$ and $\beta$ then its characteristic function is given by

$$
\frac{1}{B(p, q)}\left(1-\frac{i t}{\alpha \beta}\right)^{-\alpha} \int_{0}^{\infty} \frac{r^{p-1}}{(1+r)^{p+q}} e^{-r \ln \left(1-\frac{i t}{\alpha \beta}\right)} d r
$$

and Mean $=\frac{1}{\alpha \beta}\left[\alpha+\frac{p}{q-1}\right]$, Variance $=\frac{1}{(\alpha \beta)^{2}}\left[\alpha+\frac{p}{q-1}+\frac{p(p+1)}{(q-1)(q-2)}-\frac{p^{2}}{(q-1)^{2}}\right]$,

$$
\begin{gathered}
\beta_{1}=\frac{\left[2 \alpha+2 \frac{p}{q-1}+3 \frac{p(p+1)}{(q-1)(q-2)}+\frac{p(p+1)(p+2)}{(q-1)(q-2)(q-3)}-3 \frac{p^{2}}{(q-1)^{2}}-3 \frac{p^{2}(p+1)}{(q-1)^{2}(q-2)}+2 \frac{p^{3}}{(q-1)^{3}}\right]^{2}}{\left[\alpha+\frac{p}{q-1}+\frac{p(p+1)}{(q-1)(q-2)}-\frac{p^{2}}{(q-1)^{2}}\right]^{3}} \\
\beta_{2}=\frac{\left.-(6 \alpha+8) \frac{p^{2}}{(q-1)^{2}}-12 \frac{p^{2}(p+1)}{(q-1)^{2}(q-2)}-4 \frac{p^{2}(p+1)(p+2)}{(q-1)^{2}(q-2)(q-3)}+6 \frac{p^{3}}{(q-1)^{3}}+6 \frac{p^{3}(p+1)}{(q-1)^{3}(q-2)}-3 \frac{p^{4}}{(q-1)^{4}}\right]}{\left[\alpha+\frac{p}{q-1}+\frac{p(p+1)}{(q-1)(q-2)}-\frac{p^{2}}{(q-1)^{2}}\right]^{2}} .
\end{gathered}
$$

Remark. If $p=q=0$ then all the values of $\phi_{x}(t), \mu_{s}^{\prime}, \mu_{1}, \mu_{2}, \mu_{3}, \mu_{4}, \beta_{1}$ and $\beta_{2}$ are true for Erlang distribution with parameters $\alpha$ and $\beta$.

Estimating parameters: For a random sample $X_{1}, X_{2}, \ldots . . X_{m}$ from the distribution (16), we assume that parameters $p, q$ and $\beta$ is known and $\alpha$ unknown parameter. Now, $\mu_{1}^{\prime}=\frac{1}{\alpha \beta}\left[\alpha+\frac{p}{q-1}\right]$ and $m_{1}^{\prime}=\frac{\sum x_{i}}{m}=\bar{X}$. We obtain $\frac{1}{\alpha \beta}\left[\alpha+\frac{p}{q-1}\right]=\bar{X}$. Therefore,

$$
\hat{\alpha}=\frac{\frac{p}{q-1}}{(\bar{X} \beta-1)}
$$

Definition 3.7. A random variable $\mathrm{X}$ having the density function

$$
f(x ; p, q, m)=\int_{0}^{\infty} \frac{1}{B(p, q)} \frac{r^{p-1}}{(1+r)^{p+q}} \frac{(r+1) x^{r}}{\mathrm{~m}^{\mathrm{r}+1}} d r ; 0<x<m,
$$

is said as beta second kind mixture of Rectangular distribution with parameters $p, q$ and $m$ since

$$
\int_{0}^{m} f(x ; p, q, m) d x=1 .
$$

Different moments of the abovementioned distribution are expressed in the theorem below. 
Theorem 3.6. If $X$ follows a beta second kind mixture of rectangular distribution with parameters $p, q$ and $m$ then its characteristic function is obtained as

$$
\int_{0}^{\infty} \frac{1}{B(p, q)} \frac{r^{p-1}}{(1+r)^{p+q}} \sum_{k=0}^{\infty} \frac{(i t)^{k}}{k !} \frac{(r+1)}{m^{r+1}} \frac{m^{r+k+1}}{r+k+1} d r
$$

and the $s^{\text {th }}$ moment about origin is

$$
m^{s} \int_{0}^{\infty} \frac{1}{B(p, q)} \frac{r^{p-1}}{(1+r)^{p+q}} \frac{r+1}{r+s+1} d r
$$

Remark. If $p=q=0$ then all the values of $\phi_{x}(t), \mu_{s}^{\prime}, \mu_{1}^{\prime}, \mu_{2}^{\prime}, \mu_{2}$, are true for Rectangular distribution with parameter $m$.

Definition 3.8. A random variable $\mathrm{X}$ having the density function

$$
f(x, p, q, \alpha, \beta)=\int_{0}^{\infty} \frac{1}{B(p, q)} \frac{r^{p-1}}{(1+r)^{p+q}} \frac{x^{\alpha+r-1}(1-x)^{\beta-1}}{\mathrm{~B}(\alpha+\mathrm{r}, \beta)} d r ; 0<x<1,
$$

is called a beta second kind mixture of Beta distribution of first kind with parameters $p, q, \alpha$ and $\beta$ since

$$
\int_{0}^{\infty} f(x ; p, q, \alpha, \beta) d x=1 .
$$

Different moments of the same distribution are provided in the following theorem.

Theorem 3.7. If $X$ follows beta second kind mixture of beta distribution of first kind with parameters $p, q, \alpha$ and $\beta$ then its $s^{\text {th }}$ moment about origin is given by

$$
\int_{0}^{\infty} \frac{1}{B(p, q)} \frac{r^{p-1}}{(1+r)^{p+q}} \frac{B(\alpha+s+r, \beta)}{B(\alpha+r, \beta)} d r
$$

Remark. If we put $p=q=0$ then all the values of $\mu_{s}^{\prime}, \mu_{1}^{\prime}, \mu_{2}^{\prime}$, and $\mu_{2}$ are true for beta distribution of first kind with parameters $\alpha$ and $\beta$.

Definition 3.8. A random variable $\chi^{2}$ with the density function

$$
f\left(\chi^{2} ; p, q, n\right)=\int_{0}^{\infty} \frac{1}{B(p, q)} \frac{r^{p-1}}{(1+r)^{p+q}} \frac{e^{-\frac{1}{2} \chi^{2}}\left(\chi^{2}\right)^{\frac{n}{2}+r-1}}{2^{\frac{n}{2}+\mathrm{r}} \Gamma\left(\frac{\mathrm{n}}{2}+\mathrm{r}\right)} d r ; \chi^{2}>0,
$$

is said to have a beta second kind mixture of chi-square distribution having the parameters $p, q$ and $n$ since

$$
\int_{0}^{\infty} f\left(\chi^{2} ; p, q, n\right) d \chi^{2}=1
$$

Some characteristics of the distribution are represented in the theorem below.

Theorem 3.7. If $\chi^{2}$ has beta second kind mixture chi-square distribution with parameters p, qand $n$ then its characteristic function is expressed as

$$
\begin{aligned}
& \frac{1}{B(p, q)}(1-2 i t)^{-\frac{n}{2}} \int_{0}^{\infty} \frac{r^{p-1}}{(1+r)^{p+q}} e^{-r \ln (1-2 i t)} d r \\
& \text { and Mean }=n+2 \frac{p}{q-1}, \text { Variance }=\left[2 n+4 \frac{p}{q-1}+4 \frac{p(p+1)}{(q-1)(q-2)}-4 \frac{p^{2}}{(q-1)^{2}}\right] \\
& \beta_{1}=\frac{\left[8 n+16 \frac{p}{q-1}+24 \frac{p(p+1)}{(q-1)(q-2)}+8 \frac{p(p+1)(p+2)}{(q-1)(q-2)(q-3)}-24 \frac{p^{2}}{(q-1)^{2}}-24 \frac{p^{2}(p+1)}{(q-1)^{2}(q-2)}+16 \frac{p^{3}}{(q-1)^{3}}\right]^{2}}{\left[2 n+4 \frac{p}{q-1}+4 \frac{p(p+1)}{(q-1)(q-2)}-4 \frac{p^{2}}{(q-1)^{2}}\right]^{3}}, \\
& {\left[12 n^{2}+48 n+(48 n+96) \frac{p}{q-1}+(48 n+176) \frac{p(p+1)}{(q-1)(q-2)}+96 \frac{p(p+1)(p+2)}{(q-1)(q-2)(q-3)}+16 \frac{p(p+1)(p+2)(p+3)}{(q-1)(q-2)(q-3)(q-4)}\right.} \\
& \beta_{2}=\frac{\left.-(48 n+128) \frac{p^{2}}{(q-1)^{2}}-192 \frac{p^{2}(p+1)}{(q-1)^{2}(q-2)}-64 \frac{p^{2}(p+1)(p+2)}{(q-1)^{2}(q-2)(q-3)}+96 \frac{p^{3}}{(q-1)^{3}}+96 \frac{p^{3}(p+1)}{(q-1)^{3}(q-2)}-48 \frac{p^{4}}{(q-1)^{4}}\right]}{\left[2 n+4 \frac{p}{q-1}+4 \frac{p(p+1)}{(q-1)(q-2)}-4 \frac{p^{2}}{(q-1)^{2}}\right]^{2}} .
\end{aligned}
$$


Remark. Putting $p=q=0$ we find that all the values of $\phi_{x}(t), \mu_{1}, \mu_{2}, \mu_{3}, \mu_{4}, \beta_{1}$ and $\beta_{2}$ are true for chi-square distribution with parameters $n$.

Estimates of parameters: Let $X_{1}, X_{2}, \ldots . . X_{m}$ be a random sample from the distribution (26). We assume that parameters $p$ and $q$ is known and $n$ is unknown. Now, $\mu_{1}^{\prime}=n+2 \frac{p}{q-1}$ and $m_{1}^{\prime}=\frac{\sum x_{i}}{m}=\bar{X}$. Hence, we get $n+2 \frac{p}{q-1}=\bar{X}$. Therefore,

$$
\hat{n}=\bar{X}-2 \frac{p}{q-1}
$$

Definition 3.9. If $t$ as a random variable has the density function

$$
f(t ; p, q, n)=\int_{0}^{\infty} \frac{1}{B(p, q)} \frac{r^{p-1}}{(1+r)^{p+q}} \frac{t^{2 r}}{\mathrm{n}^{\frac{1}{2}+\mathrm{r}} \mathbf{B}\left(\frac{1}{2}+\mathrm{r}, \frac{\mathrm{n}}{2}\right)\left(1+\frac{\mathrm{t}^{2}}{\mathrm{n}}\right)^{\frac{\mathrm{n}+1}{2}+\mathrm{r}}} d r ;-\infty<t<\infty,
$$

then it is said to have a beta second kind mixture of $t$ distribution with parameters $p, q$ and $n$ provided

$$
\int_{-\infty}^{\infty} f(t ; p, q, n) d t=1
$$

The following theorem expresses here some of the properties of the distribution.

Theorem 3.8. If $t$ is beta second kind mixture of $t$ distribution with parameters $p$, qand $n$ then the $2 s^{\text {th }}$ moment about origin is given by

$$
n^{s} \int_{0}^{\infty} \frac{1}{B(p, q)} \frac{r^{p-1}}{(1+r)^{p+q}} \frac{\Gamma\left(r+s+\frac{1}{2}\right) \Gamma\left(\frac{n}{2}-s\right)}{\Gamma\left(\frac{1}{2}+r\right) \Gamma\left(\frac{n}{2}\right)} d r
$$

and the $(2 s+1)^{\text {th }}$ moment about origin is zero. $\beta_{1}=0, \beta_{2}=\frac{n-2}{n-4} \frac{\left[3+8 \frac{p}{q-1}+4 \frac{p(p+1)}{(q-1)(q-2)}\right]}{\left[1+2 \frac{p}{q-1}\right]^{2}}$.

Remark. If $p=q=0$ then all the values of $\mu_{2 s+1}, \mu_{2 s}, \mu_{1}, \mu_{2}, \mu_{3}, \mu_{4}, \beta_{1}$ and $\beta_{2}$ are true for $\mathrm{t}$ distribution with parameter $n$.

Definition 3.10. A random variable $F$ having the density function

$$
f\left(F ; p, q, n_{1}, n_{2}\right)=\int_{0}^{\infty} \frac{1}{B(p, q)} \frac{r^{p-1}}{(1+r)^{p+q}} \frac{\left(\frac{n_{1}}{n_{2}}\right)^{\frac{n_{1}}{2}+r} F^{\frac{n_{1}}{2}+r-1}}{\mathbf{B}\left(\frac{n_{1}}{2}+\mathrm{r}, \frac{\mathrm{n}_{2}}{2}\right)\left(1+\frac{n_{1}}{n_{2}} \mathrm{~F}\right)^{\frac{\mathrm{n}_{1}+\mathrm{n}_{2}}{2}+\mathrm{r}}} d r ; F>0,
$$

is said to have a beta second kind mixture of $F$ distribution with parameters $p, q, n_{1}$ and $n_{2}$ since

$$
\int_{0}^{\infty} f\left(F ; p, q, n_{1}, n_{2}\right) d F=1
$$

The following theorem presents the characteristic function and moments of this distribution.

Theorem 3.9. If F follows beta second kind mixture of $F$ distribution with parameters $p, q, n_{1}$ and $n_{2}$ then the its characteristic function is given by

$$
\int_{0}^{\infty} \frac{1}{B(p, q)} \frac{r^{p-1}}{(1+r)^{p+q}} \sum_{x=0}^{\infty} \frac{\left(i t \frac{n_{2}}{n_{1}}\right)^{x}}{x !} \frac{\Gamma\left(\frac{n_{1}}{2}+r+x\right) \Gamma\left(\frac{n_{2}}{2}-x\right)}{\Gamma\left(\frac{n_{1}}{2}+r\right) \Gamma\left(\frac{n_{2}}{2}\right)}
$$

and the $s^{\text {th }}$ moment about origin is

$$
\left(\frac{n_{2}}{n_{1}}\right)^{s} \int_{0}^{\infty} \frac{1}{B(p, q)} \frac{r^{p-1}}{(1+r)^{p+q}} \frac{\Gamma\left(\frac{n_{1}}{2}+r+s\right) \Gamma\left(\frac{n_{2}}{2}-s\right)}{\Gamma\left(\frac{n_{1}}{2}+r\right) \Gamma\left(\frac{n_{2}}{2}\right)} d r
$$

Then, Mean $=\frac{n_{2}^{2}}{n_{1}^{2}\left(n_{2}-2\right)\left(n_{2}-4\right)}\left[n_{1}\left(n_{1}+2\right)+4\left(n_{1}+1\right) \frac{p}{q-1}+4 \frac{p(p+1)}{(q-1)(q-2)}\right]$

Variance $=\frac{n_{2}^{2}}{n_{1}^{2}\left(n_{2}-2\right)\left(n_{2}-4\right)}\left[n_{1}\left(n_{1}+2\right)+4\left(n_{1}+1\right) \frac{p}{q-1}+4 \frac{p(p+1)}{(q-1)(q-2)}\right]-\left[\frac{n_{2}}{n_{1}\left(n_{2}-2\right)}\left\{n_{1}+2 \frac{p}{q-1}\right\}\right]^{2}$.

Remark. If $p=q=0$ then all the values of $\phi_{x}(t), \mu_{s}^{\prime}, \mu_{1}^{\prime}, \mu_{2}^{\prime}$ and $\mu_{2}$, are true for $F$ distribution with parameters $n_{1}$ and $n_{2}$. 


\section{CONCLUSION}

Various integral representations of beta functions can be found using the mixture beta distributions. These integrals are useful in finding mathematical and statistical properties of the various beta behavioral populations. A comparison among various features of the different beta second kind mixture distributions is shown in the tables of Appendix.

\section{CONFLICTS OF INTEREST}

Author has no conflicts of interest.

\section{REFERENCES}

1. N.L. Jonson, S. Kortz, A.W. Kemp, Univariate Discrete Distributions, second ed., Wiley, New York, NY, USA, 1992.

2. N.L. Jonson, S. Kortz, N. Balakrishnan, Continuous Univariate Distributions, second ed., vol. 1 and 2, Wiley, New York, NY, USA, 2004.

3. K. Pearson, Phil. Trans. Roy. Soc. A. 185 (1894), 71-110.

4. P.R. Rider, Ann. Math. Stat. 32 (1961), 143-147.

5. W.R. Blichke, in: G.P. Patil (ed.), Mixtures of Distribution in Classical and Contagious Discrete Distributions, Calcutta Statistical Publishing Society, 1963, pp. 351-373.

6. W.R. Blischke, Ann. Math. Stat. 33 (1962), 444-454.

7. W.R. Blischke, in: C.P. Patil (ed.), Classical and Contagious Discrete Distributions, Calcutta Statistical Publishing Society, 1963 , pp. $351-373$.

8. W.R. Blischke, J. Am. Stat. Assoc. 59 (1964), 510-528.

9. A.C. Cohen, in: Proceedings of the International Symposium on Discrete Distributions, Montreal, Canada, 1963, pp. 373-378.

10. J. Chahine, Revue De Statistiche Appliquee. 13 (1965), 33-43.

11. V. Hasselblad, Technometrics. 8 (1966), 431-444.

12. N.E. Day, Biometrika. 56 (1969), 463-474.

13. N.P. Jewell, Ann. Stat. 10 (1982), 479-484.

14. M.K. Roy, S. Rahman, M.M. Ali, J. Inf. Optim. Sci. 13 (1992), 207-208.

15. M.K. Roy, et al., J. Inf. Optim. Sci. 19 (1994), 285-302.

16. M.K. Roy, S.K. Sinha, Metron. 3 (1995), 83-92.

17. M.A.S. Adnan, H. Kiser, Far East J. Appl. Math. 94 (2016), 157-171.

18. M.A.S. Adnan, H. Kiser, J. Interdiscip. Math. 17 (2015), 403-422.

19. M.A.S. Adnan, et al., Far East J. Appl. Math. 76 (2013), 25-38.

20. M.A.S. Adnan, H. Kiser, M. Shamsuddin, Far East J. Appl. Math. 74 (2012), 49-70.

21. M.A.S. Adnan, H. Kiser, J. Biomet. Biostat. 3 (2012), 1-6.

22. M.A.S. Adnan, H. Kiser, in: Proceedings of 2011 Joint Statistical Meetings (JSM 2011), American Statistical Association, Florida, FL, USA, 2011, pp. 2336-2347.

23. M.A.S. Adnan, H. Kiser, J. Interdiscip. Math. 14 (2011), 507-522.

24. M.A.S. Adnan, H. Kiser, in: Proceedings of 2010 Joint Statistical Meetings (JSM 2010), American Statistical Association, Vancouver, Canada, 2010, pp. 2982-2996.

25. M.A.S. Adnan, H. Kiser, in: Proceedings of 2010 Joint Statistical Meetings (JSM 2010), American Statistical Association, Vancouver, Canada, 2010, pp. 4247-4261.

26. M.A.S. Adnan, H. Kiser, J. Appl. Stat. Sci. 17 (2009), 549-560.

27. M.A.S. Adnan, H. Kiser, A.S. Adnan, S. Shamsi, Far East J. Theor. Stat. 60 (2020), 1-23.

28. M.A.S. Adnan, H. Kiser, Far East J. Theor. Stat. 59 (2020), 59-79. 


\section{APPENDIX}

A comparison among various features of the different beta second kind mixtured distributions is shown in the following Tables A1 and A2.

Table A1 Comparison of density functions of different beta second kind mixture distributions.

\section{Sl. Name of the Distribution}

$1 \quad$ Beta 2nd kind mixture normal
Beta 2nd kind mixture lognormal

Beta 2nd kind mixture gamma

Beta 2nd kind mixture exponential

Beta 2nd kind mixture Erlang

Beta 2nd kind mixture rectangular

Beta 2nd kind mixture beta 1st kind

Beta 2nd kind mixture chi-square

Beta 2nd kind mixture $t$

\section{Probability Density Function $f(x)$}

$$
\int_{0}^{\infty} \frac{1}{B(p, q)} \frac{r^{p-1}}{(1+r)^{p+q}} \frac{e^{-\frac{1}{2} x^{2}} x^{2 r}}{2^{\mathrm{r}+\frac{1}{2}} \Gamma\left(\mathrm{r}+\frac{1}{2}\right)} d r
$$

Support

Parameters

$$
\int_{0}^{\infty} \frac{1}{B(p, q)} \frac{r^{p-1}}{(1+r)^{p+q}} \frac{e^{-\frac{1}{2}(\log x)^{2}}(\log x)^{2 r}}{x 2^{\mathrm{r}+\frac{1}{2}} \Gamma\left(\mathrm{r}+\frac{1}{2}\right)} d r
$$$$
\int_{0}^{\infty} \frac{1}{B(p, q)} \frac{r^{p-1}}{(1+r)^{p+q}} \frac{\beta^{\alpha+r} e^{-\beta x} x^{\alpha+r-1}}{\Gamma(\alpha+\mathrm{r})} d r
$$

$x>0$

$x>0$

$x>0$

$x>0$

$0<x<m$

$0<x<1$

$\chi^{2}>0$

$-\infty<t<\infty$

$\int_{0}^{\infty} \frac{1}{B(p, q)} \frac{r^{p-1}}{(1+r)^{p+q}} \frac{t^{2 r}}{\mathrm{n}^{\frac{1}{2}+\mathrm{r}} \mathbf{B}\left(\frac{1}{2}+\mathrm{r}, \frac{\mathrm{n}}{2}\right)\left(1+\frac{\mathrm{t}^{2}}{\mathrm{n}}\right)^{\frac{\mathrm{n}+1}{2}+\mathrm{r}}} d r$

$\int_{0}^{\infty} \frac{1}{B(p, q)} \frac{r^{p-1}}{(1+r)^{p+q}} \frac{\left(\frac{n_{1}}{n_{2}}\right)^{\frac{n_{1}}{2}+r} F^{\frac{n_{1}}{2}+r-1}}{\mathbf{B}\left(\frac{n_{1}}{2}+\mathrm{r}, \frac{\mathrm{n}_{2}}{2}\right)\left(1+\frac{n_{1}}{n_{2}} \mathrm{~F}\right)^{\frac{\mathrm{n}_{1}+\mathrm{n}_{2}}{2}+\mathrm{r}}} d r$ $p, q, n$

$p, q$

$p, q$

$p, q, \alpha, \beta$

$p, q, \alpha$

$p, q, \alpha, \beta$

$p, q, m$

$p, q, \alpha, \beta$

$p, q, n$

$p, q, n_{1}, n_{2}$

\begin{tabular}{|c|c|c|c|}
\hline Sl. & Name of the Distribution & Mean & Variance \\
\hline 1 & Beta 2nd kind mixture normal & 0 & $1+2 \frac{p}{q-1}$ \\
\hline 2 & Beta 2nd kind mixture lognormal & Can be obtained from Equation (7) & Can be obtained from Equation (7) \\
\hline 3 & Beta 2nd kind mixture gamma & $\frac{1}{\beta}\left[\alpha+\frac{p}{q-1}\right]$ & $\frac{1}{\beta^{2}}\left[\alpha+\frac{p}{q-1}+\frac{p(p+1)}{(q-1)(q-2)}-\frac{p^{2}}{(q-1)^{2}}\right]$ \\
\hline 4 & Beta 2nd kind mixture exponential & $\frac{1}{\alpha}\left[1+\frac{p}{q-1}\right]$ & $\frac{1}{\alpha^{2}}\left[1+\frac{p}{q-1}+\frac{p(p+1)}{(q-1)(q-2)}-\frac{p^{2}}{(q-1)^{2}}\right]$ \\
\hline 5 & Beta 2nd kind mixture Erlang & $\frac{1}{\alpha \beta}\left[\alpha+\frac{p}{q-1}\right]$ & $\frac{1}{(\alpha \beta)^{2}}\left[\alpha+\frac{p}{q-1}+\frac{p(p+1)}{(q-1)(q-2)}-\frac{p^{2}}{(q-1)^{2}}\right]$ \\
\hline 6 & Beta 2nd kind mixture rectangular & Can be achieved from Equation (22) & Can be achieved from Equation (22) \\
\hline 7 & Beta 2nd kind mixture beta 1st kind & Equation (25) provides & Equation (25) provides \\
\hline 8 & Beta 2nd kind mixture chi-square & $n+2 \frac{p}{q-1}$ & {$\left[2 n+4 \frac{p}{q-1}+4 \frac{p(p+1)}{(q-1)(q-2)}-4 \frac{p^{2}}{(q-1)^{2}}\right]$} \\
\hline 9 & Beta 2nd kind mixture $t$ & 0 & $\frac{n}{n-2}\left[1+2 \frac{p}{q-1}\right]$ \\
\hline 10 & Beta 2nd kind mixture $F$ & $\begin{array}{l}\frac{n_{2}^{2}}{n_{1}^{2}\left(n_{2}-2\right)\left(n_{2}-4\right)} \\
{\left[n_{1}\left(n_{1}+2\right)+4\left(n_{1}+1\right) \frac{p}{q-1}+4 \frac{p(p+1)}{(q-1)(q-2)}\right]}\end{array}$ & $\begin{array}{l}\frac{n_{2}^{2}}{n_{1}^{2}\left(n_{2}-2\right)\left(n_{2}-4\right)}\left[n_{1}\left(n_{1}+2\right)+4\left(n_{1}+1\right) \frac{p}{q-1}\right. \\
\left.+4 \frac{p(p+1)}{(q-1)(q-2)}\right]-\left[\frac{n_{2}}{n_{1}\left(n_{2}-2\right)}\left\{n_{1}+2 \frac{p}{q-1}\right\}\right]^{2}\end{array}$ \\
\hline
\end{tabular}

Table A2 Comparison among first two moments of different beta second kind mixture distributions.

Comments If $\alpha=1$, then the beta 2nd kind mixture of gamma distribution and Beta 2nd kind mixture of Erlang distribution becomes Beta 2nd kind mixture of exponential distribution. 